\title{
A four-year baseline Swift study of enigmatic X-ray transients located near the Galactic center
}

\author{
N. Degenaar and R. Wijnands \\ University of Amsterdam, Postbus 94249, 1098 SJ Amsterdam, The Netherlands \\ e-mail: degenaar@uva.nl
}

Received 1 July 2010 / Accepted 21 September 2010

\begin{abstract}
We report on continued monitoring observations of the Galactic center carried out by the X-ray telescope aboard the Swift satellite in 2008 and 2009. This campaign revealed activity of the five known X-ray transients AX J1745.6-2901, CXOGC J174535.5-290124, GRS 1741-2853, XMM J174457-2850.3 and CXOGC J174538.0-290022. All these sources are known to undergo very faint X-ray outbursts with 2-10 keV peak luminosities of $L_{X \text {,peak }} \sim 10^{34-36} \mathrm{erg} \mathrm{s}^{-1}$, although the two confirmed neutron star low-mass X-ray binaries AX J1745.6-2901 and GRS 1741-2853 can also become brighter $\left(L_{\mathrm{X} \text {,peak }} \sim 10^{36-37} \mathrm{erg} \mathrm{s}^{-1}\right)$. We discuss the observed longterm lightcurves and X-ray spectra of these five enigmatic transients. In 2008, AX J1745.6-2901 returned to quiescence following an unusually long accretion outburst of more than 1.5 years. GRS 1741-2853 was active in 2009 and displayed the brightest outburst ever recorded for this source, reaching up to a $2-10 \mathrm{keV}$ luminosity of $L_{\mathrm{X}} \sim 1 \times 10^{37}(D / 7.2 \mathrm{kpc})^{2} \mathrm{erg} \mathrm{s}^{-1}$. This system appears to undergo recurrent accretion outbursts approximately every 2 years. Furthermore, we find that the unclassified transient XMM J174457-2850.3 becomes bright only during short episodes (days) and is often found active in between quiescence $\left(L_{\mathrm{X}} \sim 10^{32} \mathrm{erg} \mathrm{s}^{-1}\right)$ and its maximum outburst luminosity of $L_{\mathrm{X}} \sim 10^{36} \mathrm{erg} \mathrm{s}^{-1}$. CXOGC J174535.5-290124 and CXOGC J174538.0-290022, as well as three other veryfaint X-ray transients that were detected by Swift monitoring observations in 2006, have very low time-averaged mass-accretion rates of $\langle\dot{M}\rangle_{\text {long }} \lesssim 2 \times 10^{-12} M_{\odot} \mathrm{yr}^{-1}$. Despite having obtained two years of new data in 2008 and 2009, no new X-ray transients were detected.
\end{abstract}

Key words. X-rays: binaries - stars: neutron - accretion, accretion disks - Galaxy: center - X-rays: general

\section{Introduction}

Starting in 2006 February, the Swift satellite has been monitoring the Galactic center (GC) with the onboard X-ray telescope (XRT, Burrows et al. 2005). In this campaign, short ( $\sim 1 \mathrm{ks})$ pointings are carried out on an almost daily basis ${ }^{1}$, covering a field of $\sim 26^{\prime} \times 26^{\prime}$ of sky around Sgr A* (Kennea \& The Swift/XRT team 2006b; Degenaar \& Wijnands 2009). This is an ideal setting for detecting transient $\mathrm{X}$-ray sources in one of the most active X-ray regions in the Milky Way.

$\mathrm{X}$-ray transients alternate periods of quiescence, that have a typical duration of years to decades and are characterized by $2-$ $10 \mathrm{keV}$ luminosities of $L_{\mathrm{X}} \sim 10^{30-33} \mathrm{erg} \mathrm{s}^{-1}$, with occasional outbursts during which the X-ray luminosity increases by a factor $\gtrsim 100$ for weeks to months. A large fraction of the galactic X-ray transients can be identified with neutron stars or black holes accreting matter from a companion star in an X-ray binary. Based on the nature of the donor star, we can distinguish lowmass X-ray binaries (LMXBs, $M_{\text {donor }} \lessgtr 1 M_{\odot}$, spectral type later than B) or high-mass X-ray binaries (HMXBs, $M_{\text {donor }} \gtrsim 10 M_{\odot}$, spectral type $\mathrm{O}$ or $\mathrm{B}$ ).

In LMXBs, matter is generally transferred because the donor star fills its Roche lobe, a process that involves the formation of an accretion disk. In such systems, the transient behavior is explained in terms of a thermal-viscous instability that causes the disk to oscillate between a cold, neutral state (quiescence), and one in which it is hot and ionized, causing a strong increase in

\footnotetext{
${ }^{1}$ Except during the months November-February, when the GC is too close (within 45 degrees) to the Sun.
}

the mass-accretion rate and resulting in an X-ray outburst (e.g., King \& Ritter 1998; Lasota 2001). During quiescence, the disk regains the mass that was lost during the outburst and the cycle repeats. Symbiotic X-ray binaries form a small sub-class of LMXBs in which the compact primary, most likely a neutron star, is accreting matter from the wind of an M-type giant companion (e.g., Masetti et al. 2007).

Amongst the transient HMXBs, most of the currently known systems are $\mathrm{Be} / \mathrm{X}$-ray binaries. In such systems, the compact primary is in a wide and eccentric orbit accreting matter from the circumstellar disk surrounding a main sequence Oe or Be star around periastron passage (e.g., Negueruela 2004). However, recently Integral and RXTE have unveiled a new class of transient HMXBs, called Supergiant Fast X-ray transients (SFXTs, e.g., Negueruela et al. 2006), in which the compact star is capturing the strong stellar wind of an $\mathrm{O}$ or $\mathrm{B}$ supergiant companion. In these systems, the transient behavior is thought to be caused by clumpy or anisotropic winds (e.g., Sidoli 2009).

The temporal and spectral properties of the brightest galactic X-ray transients, which have $2-10 \mathrm{keV}$ peak luminosities of $L_{\mathrm{X} \text {, peak }} \sim 10^{36-39} \mathrm{erg} \mathrm{s}^{-1}$, are well established through the work of numerous past and present X-ray missions. However, much less is known about transient sources that manifest themselves with lower 2-10 keV peak luminosities of $\sim 10^{34-36} \mathrm{erg} \mathrm{s}^{-1}$ (e.g., Sidoli et al. 1999; Muno et al. 2005; Porquet et al. 2005; Wijnands et al. 2006a; Campana 2009; Heinke et al. 2009). It is only with the advent of the current generation of sensitive X-ray instruments that the properties of such objects can be studied in detail. To date, a few tens of low-luminosity transients are known 

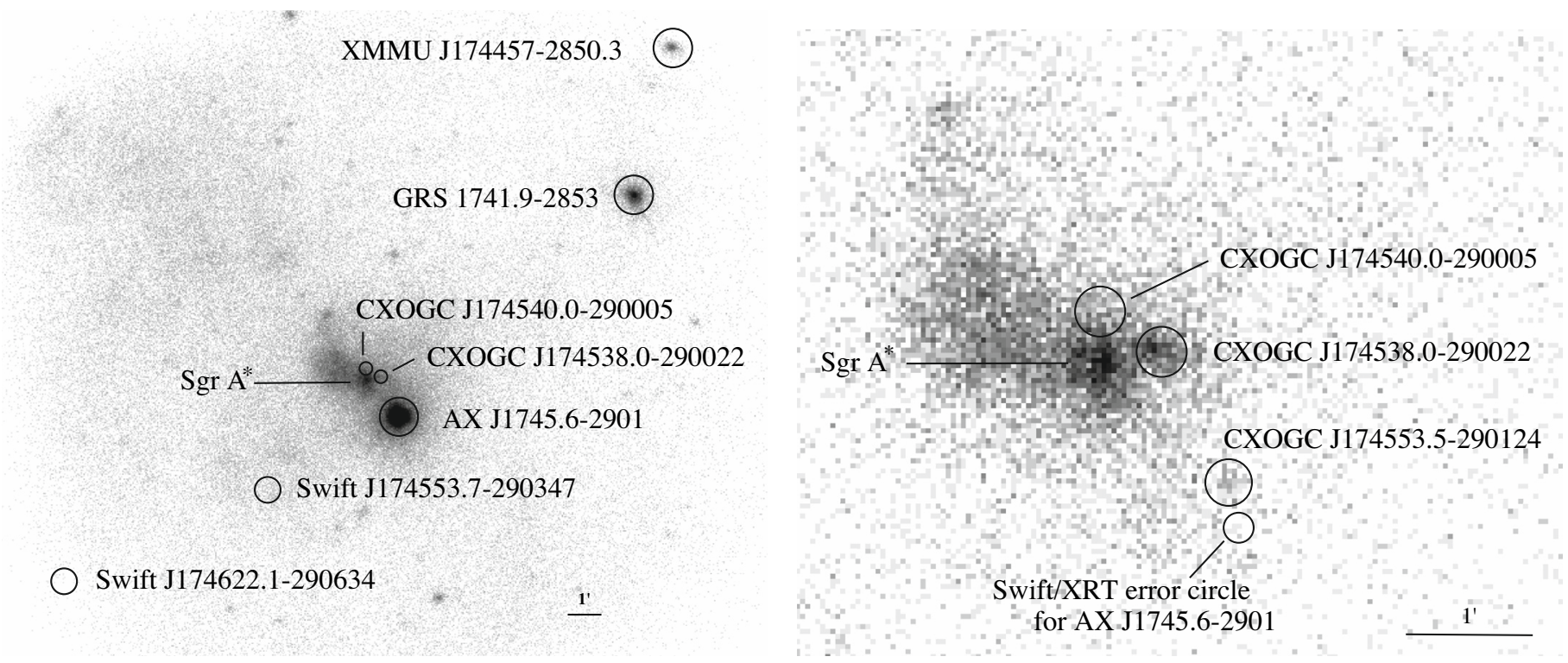

Fig. 1. Swift/XRT PC mode images $(0.3-10 \mathrm{keV})$ of the region around Sgr A*. The images indicate the locations of the five X-ray transients that were in outburst in 2008-2009, as well as three additional transients that were detected with Swift in 2006 (CXOGC J174540.0-290005, Swift J174553.7-290347 and Swift J174622.1-290634). (Left) Merged X-ray image of the data obtained in 2008 and 2009. (Right) A magnified image of the inner region around Sgr A* from the epoch between 2008 September 2 and 2009 November 1, during which AX J1745.6-2901 resided in quiescence and activity from CXOGC J174535.5-290124 could be detected. CXOGC J174538.0-290022 was also detected in outburst during that time.

in our Galaxy. As for the brighter systems, many of these are expected to harbor accreting neutron stars or black holes, but their nature and the underlying mechanism producing their subluminous outbursts is not understood well.

The hypothesis that a significant fraction of the lowluminosity transients are X-ray binaries, gains credence by the detection of thermonuclear X-ray bursts from several of these systems (e.g., in 't Zand et al. 1991; Maeda et al. 1996; Cocchi et al. 1999; Cornelisse et al. 2002; Chelovekov \& Grebenev 2007; Del Santo et al. 2007; Wijnands et al. 2009). This establishes that these objects harbor accreting neutron stars, most likely in an LMXB configuration. The observed low X-ray luminosities in combination with estimates of their recurrence times, suggest that these systems have very low time-averaged massaccretion rates (e.g., Degenaar \& Wijnands 2009). This might pose a challenge to explain the existence of these LMXBs without having to invoke exotic evolutionary scenarios (e.g., King \& Wijnands 2006).

Many low-luminosity transients are located in the vicinity of Sgr A* (e.g., Muno et al. 2005; Wijnands et al. 2006a; Degenaar $\&$ Wijnands 2009). The Swift/GC monitoring program thus provides an excellent setting to detect new low-luminosity transients and to study the long-term behavior of known systems. This opens up the possibility to gain more insight into their duty cycles and the energetics of their outbursts, and thereby to refine estimates of their average mass-accretion rates. This is an important parameter for understanding their evolution (e.g., King \& Wijnands 2006), as well as the properties of thermonuclear X-ray bursts occurring at low accretion luminosities (e.g., in 't Zand et al. 2005; Peng et al. 2007; Cooper \& Narayan 2007).

In a previous paper, we discussed a total of seven transients that were found active during Swift/XRT monitoring observations of the GC, carried out in 2006 and 2007 (Degenaar \& Wijnands 2009). Here, we discuss the data accumulated over the years 2008 and 2009, which revealed activity of five previously known X-ray transients.

\section{Observations and data analysis}

We obtained all 2008 and 2009 XRT observations of the GC from the Swift public data archive. For 2008, the nearly daily coverage resulted in a total of 171 observations, amounting to $211 \mathrm{ks}$ of exposure. All data was obtained in the photon counting (PC) mode and covers the epoch 2008 February 19October 30. In 2009, the campaign was carried out in a slightly different setting, with $\sim 1$ ks observations performed once every $\sim 3$ days, instead of the daily repetition between 2006 and 2008. Swift targeted the GC from 2009 June 4 till November 1 during 40 observations for a total exposure time of $45 \mathrm{ks}$. Most of the 2009 data was obtained in the PC mode, apart from a selection of 3 pointings, during which the XRT was operated in the windowed timing (WT) mode. These WT observations were carried out between 2009 October 10-13, and aimed specifically for GRS 1741-2853, which was in outburst at that time and caused significant pile-up in the PC data.

All raw data were processed with the XRTPIPELINE using standard quality cuts and event grades $0-12$ for the PC data and 0-2 for the WT mode observations. Figure 1 displays two accumulated X-ray images of the Swift/XRT observations. Both images clearly show regions of diffuse emission around $\mathrm{Sgr} \mathrm{A}^{*}$, as well as several X-ray point sources. Five of these can be identified with the known X-ray transients AX J1745.6-2901, CXOGC J174535.5-290124, GRS 1741-2853, XMM J1744572850.3 and CXOGC J174538.0-290022, while others are known persistent X-ray sources. In 2006, Swift detected three additional X-ray transients in outburst, which are not found active in the new 2008-2009 data set: CXOGC J174540.0290005, Swift J174553.7-290347 and Swift J174622.1-290634 (Degenaar \& Wijnands 2009). The locations of these transients are also indicated in Fig. 1. We used XSELECT (v. 2.4) to compare sub-sets of the data to determine when the transients were active. A source was considered in quiescence when it was not detected by visual inspection upon summing multiple observations. A non-detection in $5 \mathrm{ks}$ of data corresponds roughly to a 
N. Degenaar and R. Wijnands: Swift monitoring of enigmatic X-ray transients
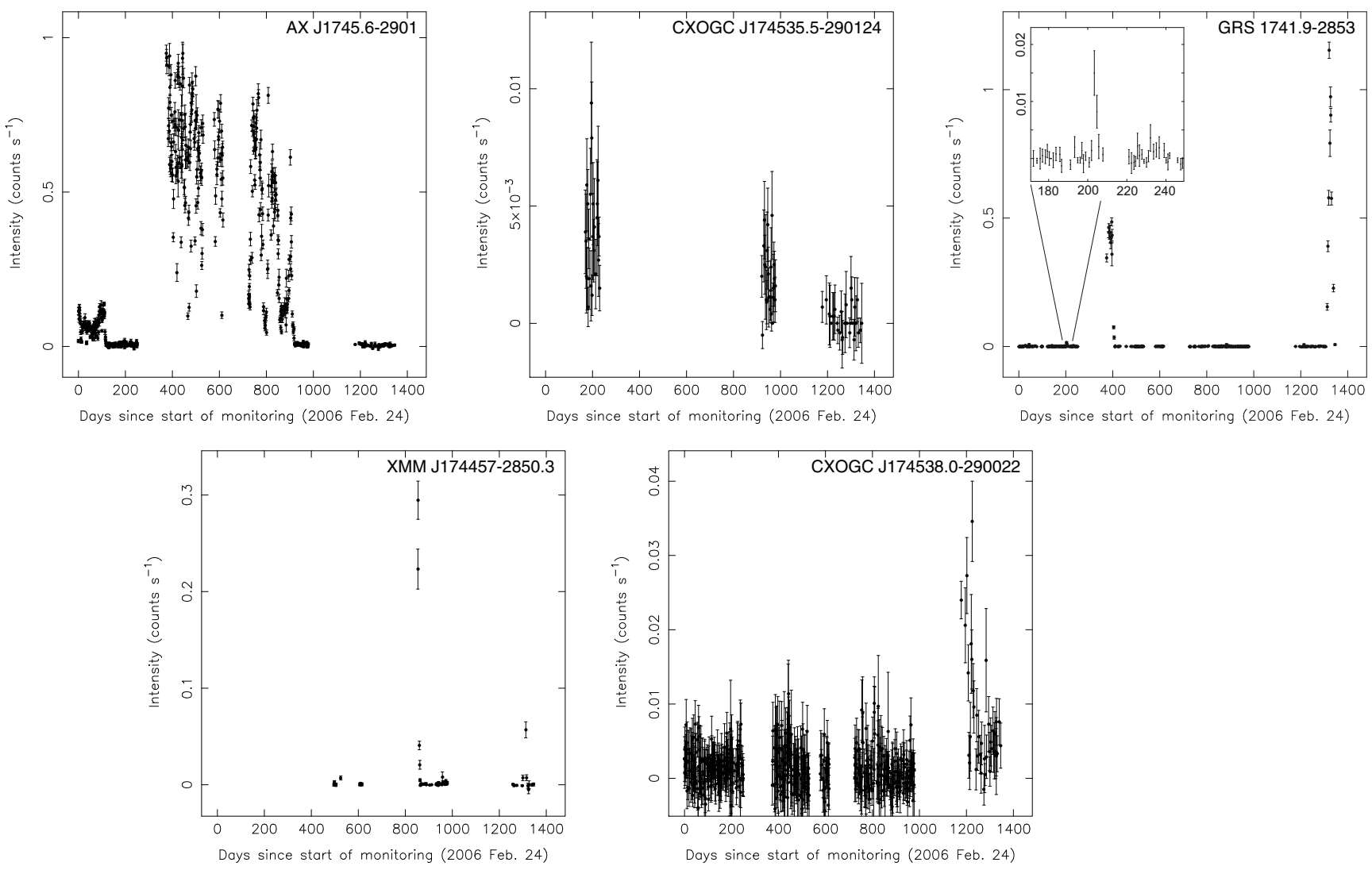

Fig. 2. Background corrected 0.3-10 keV Swift/XRT lightcurves of the five transients that were active in 2008-2009 (PC mode data only). Displayed is their long-term behavior from the start of the monitoring campaign of the GC on 2006 February 24. Days 0-616 cover the years 2006 and 2007 (discussed in Degenaar \& Wijnands 2009), whereas days 725-1346 represent the new data assembled in 2008-2009. The lightcurve of GRS 1741-2853 shows a magnified image of the short and weak outburst that occurred in 2006.

2-10 keV unabsorbed flux threshold of $\sim 2 \times 10^{-13} \mathrm{erg} \mathrm{cm}^{-2} \mathrm{~s}^{-1}$, or a luminosity of $\sim 2 \times 10^{33}(D / 8 \mathrm{kpc})^{2} \mathrm{erg} \mathrm{s}^{-1}$, although the exact value depends on the assumed spectral model.

We constructed X-ray lightcurves for the five active transients using all PC mode observations in which a source was in field of view (FOV). A circular source region with a 5 pixel radius was employed for CXOGC J174535.5-290124, XMM J174457-2850.3 and CXOGC J174538.0-290022, while a 10 pixel radius was used for the brighter AX J1745.6-2901 and GRS 1741-2853. Corresponding background events were averaged over a set of three nearby areas having the same shape and size as the source region. Particularly for sources within close proximity of Sgr $\mathrm{A}^{*}$, which are embedded in diffuse emission (see Fig. 1), the background regions were carefully chosen to account for the enhanced background level. For AX J1745.6-2901, GRS 1741-2853 and CXOGC J174538.0290022 we could use the full 2008-2009 data set to create the X-ray lightcurve. However, for both CXOGC J174535.5290124 (which is only visible when AX J1745.6-2901 is quiescent) and XMM J174457-2850.3 (which has a large offset from Sgr A* and was therefore not always in FOV), the lightcurve was constructed from a sub-set of the observations. We combined the 2008-2009 data with that of 2006-2007 (discussed in Degenaar \& Wijnands 2009), to obtain lightcurves with a four-year long baseline for the five active transients. These are displayed in Fig. 2.

The PC data of AX J1745.6-2901 and GRS 1741-2853 are subject to pile-up. This effect becomes an issue for PC mode count rates above $\sim 0.5$ counts $\mathrm{s}^{-1}$ and causes multiple photons to be registered as single events, thus underestimating the true count rate. The lightcurves of AX J1745.6-2901 and GRS 17412853 are not corrected for this. To estimate to which extend the count rates are affected, we subtracted source events from both circular and annular regions for the piled-up data of both sources. By comparing the fluxes deduced from spectral fitting, we find that pile-up causes the PC mode count rates to be underestimated by a factor of $\sim 1.2$ at $\sim 0.7$ counts $\mathrm{s}^{-1}, \sim 1.5$ at $\sim 1.0$ counts $\mathrm{s}^{-1}$ (about the peak count rate of AX J1745.6-2901) and $\sim 3$ at $\sim 1.8$ counts $^{-1}$ (the peak count rate observed for GRS 17412853).

We combined all the pointings in which a source was active to create a summed outburst spectrum (displayed in Fig. 3). For AX J1745.6-2901 and GRS 1741-2853, we attempted to circumvent the effect of pile-up on the spectral shape by using an annular extraction region for the PC data, following the Swift pile-up analysis thread ${ }^{2}$. As such, we used an annulus with an inner (outer) radius of 6 (15) pixels to extract source events from AX J1745.6-2901, and an inner (outer) radius of 7 (30) pixels for GRS 1741-2853. We compared the pile-up corrected PC spectra of GRS 1741-2853 with spectra obtained from quasisimultaneous WT mode data, which are not subject to pile-up. For the WT data, we used a $40 \times 40$ pixels rectangular extraction region for source events, and a box of similar dimensions as a background reference. From spectral fitting, we obtained fluxes for the different modes that differ by $\sim 10 \%$, and spectral parameters that are consistent with one another within the errors. This

${ }^{2}$ http://www.swift.ac.uk/pileup.shtml 

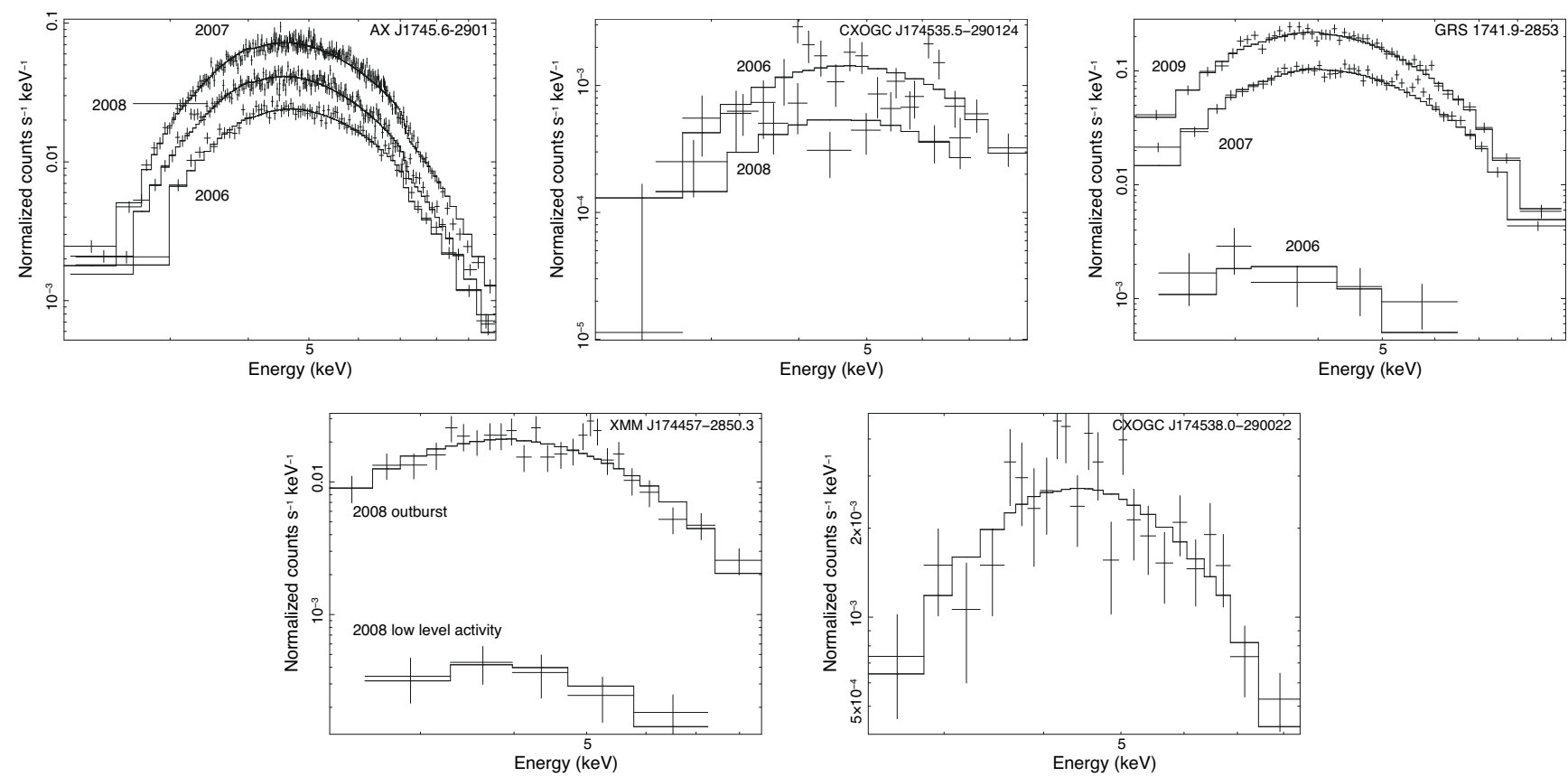

Fig. 3. Background corrected Swift/XRT average outburst spectra. For AX J1745.6-2901, CXOGC J174535.5-290124 and GRS 1741-2853, spectra of the different outbursts captured during the 2006-2009 Swift/XRT monitoring are plotted together. The plot of XMM J174457-2850.3 shows the average outburst spectrum of the source in 2008, as well as the spectrum of the low-level activity observed in the months following this outburst.

suggests that the above described pile-up correction for the PC mode works satisfactory.

Using the software tool XRTEXPOMAP, we generated exposure maps for each observation, which carry information about the bad columns and hence the effective area of the CCD. These were subsequently used to create ancillary response files (arf) for all spectra with the task XRTMKARF. These account for different extraction regions, vignetting and corrections for the point spread function. The latest response matrix files ( $\mathrm{rmf}, \mathrm{v} .11$ ) were taken from the CALDB database. Using GRPPHA, the spectra were grouped to contain at least 20 photons per bin. However, for XMM J174457-2850.3 and CXOGC J174535.5-290124 we use bins with a minimum of 10 photons, because of the low number of counts collected for these two sources.

We fitted the average outburst spectra using XSPEC (v. 12.5, Arnaud 1996) to an absorbed powerlaw model and deduce the absorbed and unabsorbed fluxes in the $2-10 \mathrm{keV}$ energy range. For the neutral hydrogen absorption, we use the PHABS model using the default XSPEC abundances and cross-sections. When a source displayed multiple outbursts, we fitted these simultaneously with the hydrogen column density tied. We include the 2006 and 2007 data (discussed in Degenaar \& Wijnands 2009) in these fits. To calculate the unabsorbed peak flux of the outbursts of AX J1745.6-2901, XMM J174457-2850.3 and GRS 17412853, we extracted a single spectrum from the observation with the highest count rate. For the former two we use PC observations, applying pile-up corrections as described above when necessary, while for the latter source WT data was available. In case of CXOGC J174535.5-290124 and CXOGC J174538.0290022, the source count rates were too low to extract a spectrum from a single observation. Therefore, we determined a count rate to flux conversion factor for these two sources by comparing the average outburst flux with the average net count rate. We then used this to estimate the unabsorbed peak flux from the observed peak count rate.
All five active X-ray transients are heavily absorbed $\left(N_{\mathrm{H}} \gtrsim\right.$ $7 \times 10^{22} \mathrm{~cm}^{-2}$; see Table 1$)$, consistent with values obtained for sources that lie close to Sgr A*. We therefore assumed source distances of $8 \mathrm{kpc}$ when calculating 2-10 keV luminosities from the unabsorbed fluxes. However, for GRS 1741-2853 we use a distance of $7.2 \mathrm{kpc}$, since this is the upper limit that has recently been inferred from the analysis of thermonuclear X-ray bursts (Trap et al. 2009). The X-ray spectra are displayed in Fig. 3 and the results of our spectral analysis are presented in Table 1 . The 2006 and 2007 data (reported by Degenaar \& Wijnands 2009), are refitted in this work.

For each observed outburst we calculate the fluence by multiplying the average unabsorbed $2-10 \mathrm{keV}$ outburst flux with the duration of the outburst. These results are presented in Table 2. Assuming that the transients are accreting systems, we additionally calculate the mass-accretion rates during outburst. Furthermore, we use the estimated duty cycles to obtain an order of magnitude approximation for their long-term mass-accretion rates (see Sect. 4.2 for further details). These results are also included in Table 2.

\section{X-ray lightcurves and spectra}

\section{1. $A X J 1745.6-2901$}

The X-ray transient AX J1745.6-2901 was detected with the ASCA satellite in 1993 October and in 1994 October, displaying $3-10 \mathrm{keV}$ luminosities of $\sim 2 \times 10^{35}$ and $\sim 9 \times 10^{35} \mathrm{erg} \mathrm{s}^{-1}$, respectively (Maeda et al. 1996). The detection of thermonuclear X-ray bursts identified the source as a neutron star LMXB and the system displays eclipses with a period of $8.4 \mathrm{~h}$, which represents the binary orbital period (Maeda et al. 1996). The likely quiescent counterpart, CXOGC J174535.6-290133, has a 2-10 keV luminosity of several times $10^{32} \mathrm{erg} \mathrm{s}^{-1}$ (Degenaar \& Wijnands 2009). 
Table 1. Results from fitting the X-ray spectral data.

\begin{tabular}{|c|c|c|c|c|c|c|c|c|c|}
\hline Source name & Year & $\begin{array}{l}N_{\mathrm{H}} \\
\left(10^{22} \mathrm{~cm}^{-2}\right)\end{array}$ & $\Gamma$ & $\begin{array}{l}\text { Red. } \chi^{2} \\
\text { (d.o.f.) }\end{array}$ & $F_{\mathrm{X}, \mathrm{abs}}$ & $F_{\mathrm{X}, \text { unabs }}$ & $F_{X, p e a k}$ & $L_{\mathrm{X}}$ & $L_{\mathrm{X}, \text { peak }}$ \\
\hline \multirow[t]{4}{*}{ AX J1745.6-2901 } & & $23.8 \pm 0.5$ & & $1.10(1427)$ & & & & & \\
\hline & 2008 & & $2.7 \pm 0.1$ & & $22.5 \pm 0.2$ & $93.2 \pm 5.5$ & 710 & 71 & 550 \\
\hline & 2007 & & $2.7 \pm 0.1$ & & $45.2 \pm 0.3$ & $187.7 \pm 7.5$ & 800 & 145 & 610 \\
\hline & 2006 & & $2.4 \pm 0.1$ & & $14.8 \pm 0.2$ & $53.3 \pm 2.2$ & 120 & 41 & 92 \\
\hline \multirow[t]{3}{*}{ CXOGC J174535.5-290124 } & & $12.0 \pm 6.9$ & & $1.23(25)$ & & & & & \\
\hline & 2008 & & $1.0_{-1.0}^{+2.2}$ & & $0.9 \pm 0.3$ & $1.4 \pm 0.6$ & 2.6 & 1.1 & 2.0 \\
\hline & 2006 & & $0.8_{-0.8}^{+1.0}$ & & $1.3 \pm 0.2$ & $2.1 \pm 0.6$ & 4.0 & 1.6 & 3.0 \\
\hline \multirow[t]{4}{*}{ GRS 1741-2853 } & & $14.0 \pm 0.7$ & & $0.99(416)$ & & & & & \\
\hline & 2009 & & $3.0 \pm 0.2$ & & $87.2 \pm 1.5$ & $283.6 \pm 2.2$ & 2200 & 176 & 1300 \\
\hline & 2007 & & $2.6 \pm 0.1$ & & $61.7 \pm 1.0$ & $174.9 \pm 1.2$ & 260 & 109 & 150 \\
\hline & 2006 & & $5.0 \pm 2.5$ & & $0.6 \pm 0.3$ & $5.0 \pm 3.7$ & 12 & 3.1 & 7.0 \\
\hline \multirow[t]{5}{*}{ XMM J174457-2850.3 } & & $7.5 \pm 2.9$ & & $0.83(29)$ & & & & & \\
\hline & 2009 & & $2.3 \pm 1.1$ & & $11.5 \pm 1.8$ & $21.3 \pm 6.4$ & 21.8 & 16 & 17 \\
\hline & 2008 & & $1.6 \pm 0.6$ & & $20.7 \pm 2.0$ & $32.4 \pm 5.4$ & 250 & 25 & 190 \\
\hline & 2008-low & & $1.8 \pm 1.4$ & & $0.5 \pm 0.2$ & $0.8 \pm 0.2$ & 3.6 & 0.6 & 2.8 \\
\hline & 2007 & $7.5 \mathrm{fix}$ & 1.8 fix & & 0.4 & 0.6 & 1.5 & 0.4 & 1.1 \\
\hline CXOGC J174538.0-290022 & 2009 & $12.8 \pm 5.9$ & $1.4 \pm 0.9$ & $1.14(21)$ & $2.7 \pm 0.5$ & $5.0 \pm 1.4$ & 21.8 & 3.8 & 17 \\
\hline
\end{tabular}

Notes. For sources that displayed multiple outbursts, we fitted the different outburst spectra simultaneously with the hydrogen column density tied. The 2006 and 2007 data (discussed by Degenaar \& Wijnands 2009) are re-fitted in this work. Fluxes and luminosities are for the 2-10 keV energy band and given in units of $10^{-12} \mathrm{erg} \mathrm{cm}^{-2} \mathrm{~s}^{-1}$ and $10^{34} \mathrm{erg} \mathrm{s}^{-1}$, respectively. $F_{\mathrm{X}, \text { abs }}$ and $F_{\mathrm{X} \text {,unabs }}$ represent the mean absorbed and unabsorbed outburst fluxes, while $F_{\mathrm{X} \text {,peak }}$ is the unabsorbed peak flux. $L_{\mathrm{X}}$ and $L_{\mathrm{X} \text {,peak }}$ are the average and peak outburst luminosity, respectively. These are calculated from the unabsorbed fluxes by adopting a distance of $7.2 \mathrm{kpc}$ for GRS 1741-2853 and $8 \mathrm{kpc}$ for all other sources. Fluxes for the 2007 activity of XMM J174457-2850.3 were deduced using PIMMS, for fixed values of $N_{\mathrm{H}}$ and $\Gamma$.

Table 2. Overview of the outburst properties and estimated (time-averaged) mass-accretion rates.

\begin{tabular}{|c|c|c|c|c|c|c|}
\hline Source & Year & $t_{\mathrm{ob}}$ & $\tilde{F}$ & Duty cycle & $\langle\dot{M}\rangle_{\mathrm{ob}}$ & $\langle\dot{M}\rangle_{\text {long }}$ \\
\hline \multirow[t]{3}{*}{ AX J1745.6-2901 } & & & & $10-30 \%$ & & $\sim(3-8) \times 10^{-11}$ \\
\hline & $2007-2008$ & $>80$ & $>7 \times 10^{-3}$ & & $\sim 3 \times 10^{-10}$ & \\
\hline & 2006 & $>16$ & $z 5 \times 10^{-4}$ & & $\sim 1 \times 10^{-10}$ & \\
\hline \multirow[t]{3}{*}{ CXOGC J174535.5-290124 } & & & & $20-50 \%$ & & $\sim(7-14) \times 10^{-13}$ \\
\hline & 2008 & $>8$ & $\gtrsim 7 \times 10^{-6}$ & & $\sim 3 \times 10^{-12}$ & \\
\hline & 2006 & $>12$ & $\gtrsim 2 \times 10^{-5}$ & & $\sim 4 \times 10^{-12}$ & \\
\hline \multirow[t]{4}{*}{ GRS 1741-2853 } & & & & $5-15 \%$ & & $\sim(2-6) \times 10^{-11}$ \\
\hline & 2009 & $4-5$ & $\sim 8 \times 10^{-4}$ & & $\sim 5 \times 10^{-10}$ & \\
\hline & 2007 & $>13$ & $\gtrsim 1 \times 10^{-3}$ & & $\sim 3 \times 10^{-10}$ & \\
\hline & 2006 & $<1$ & $\$ 3 \times 10^{-6}$ & & $\sim 8 \times 10^{-12}$ & \\
\hline \multirow[t]{5}{*}{ XMM J174457-2850.3 } & & & & $5-50 \%$ & & $\sim(1-10) \times 10^{-12}$ \\
\hline & 2009 & $<2$ & $\lesssim 3 \times 10^{-5}$ & & $\sim 4 \times 10^{-11}$ & \\
\hline & 2008 & $1-7$ & $\sim(2-10) \times 10^{-5}$ & & $\sim 6 \times 10^{-11}$ & \\
\hline & 2008-low & $>16$ & $z 3 \times 10^{-5}$ & & $\sim 2 \times 10^{-12}$ & \\
\hline & 2007 & $<12$ & $\lesssim 4 \times 10^{-6}$ & & $\sim 1 \times 10^{-12}$ & \\
\hline CXOGC J174538.0-290022 & 2009 & $30-52$ & $\sim(9-20) \times 10^{-5}$ & $5-20 \%$ & $\sim 1 \times 10^{-11}$ & $\sim(5-20) \times 10^{-13}$ \\
\hline CXOGC J174540.0-290005 & 2006 & 2 & $\sim 1 \times 10^{-5}$ & $1-5 \%$ & $\sim 2 \times 10^{-11}$ & $\sim(3-13) \times 10^{-13}$ \\
\hline Swift J174553.7-290347 & 2006 & 2 & $\sim 8 \times 10^{-6}$ & $\$ 5 \%$ & $\sim 2 \times 10^{-11}$ & $\$ 6 \times 10^{-13}$ \\
\hline Swift J174622.1-290634 & 2006 & 5 & $\sim 5 \times 10^{-6}$ & $\lessgtr 14 \%$ & $\sim 3 \times 10^{-12}$ & $\$ 4 \times 10^{-13}$ \\
\hline
\end{tabular}

Notes. The outburst duration, $t_{\mathrm{ob}}$, is expressed in weeks. $\tilde{F}$ represents the fluence of the outburst in units of erg $\mathrm{cm}^{-2}$ in the $2-10 \mathrm{keV}^{\mathrm{anergy}} \mathrm{band}$. $\langle\dot{M}\rangle_{\mathrm{ob}}$ is the estimated average accretion rate during outburst $\left(M_{\odot} \mathrm{yr}^{-1}\right)$ assuming a neutron star primary with $M=1.4 M_{\odot}$ and $R=10 \mathrm{~km}$. The

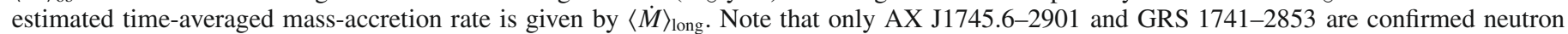
star X-ray binaries, the other six sources have an unknown nature.

Since 1994, AX J1745.6-2901 was never reported in outburst again, despite regular monitoring of the GC, e.g., with the Chandra satellite between 1999 and 2004 (Muno et al. 2003b, 2004, 2005). However, the source was found active in 2006 February, when the Swift/XRT monitoring observations of the GC kicked off (Kennea et al. 2006a) ${ }^{3}$. AX J1745.6-2901 remained active for 16 weeks at an average $2-10 \mathrm{keV}$ luminosity

\footnotetext{
3 The transient was first denoted as Swift J174535.5-290135, but the detection of $8.4 \mathrm{~h}$ eclipses in XMM-Newton observations definitely
}

of $\sim 4 \times 10^{35} \mathrm{erg} \mathrm{s}^{-1}$ (Degenaar \& Wijnands 2009; see also Table 1). Subsequently, the source resided in quiescence for at least four months (2006 July-October), but was again detected in outburst by Integral and Swift on 2007 February 1517 (Kuulkers et al. 2007a; Wijnands et al. 2007). The activity continued throughout the 2007 monitoring campaign, which ended on 2007 November 2. The average outburst luminosity

linked Swift J174535.5-290135 to AX J1745.6-2901 (Porquet et al. 2007). 
was $\sim 1.5 \times 10^{36} \mathrm{erg} \mathrm{s}^{-1}(2-10 \mathrm{keV})$, i.e., $\sim 4$ times higher than the level observed in 2006 (Porquet et al. 2007; Degenaar \& Wijnands 2009).

When the Swift/GC monitoring observations resumed on 2008 February 19, AX J1745.6-2901 was detected at a similar intensity as measured in 2007 November (see Fig. 2). This makes it likely that the outburst continued during the time that Swift could not observe the GC due to Sun-angle constraints. In 2008, the source flux was observed to decrease gradually (see Fig. 2). In late-August, the decay accelerated and within two weeks the source luminosity dropped from $\sim 10^{36} \mathrm{erg} \mathrm{s}^{-1}$ (2$10 \mathrm{keV})$, down to the background level on 2008 September 2. AX J1745.6-2901 was not detected for the remainder of the Swift/XRT observations in 2008 and the system had thus returned to quiescence following an accretion outburst that lasted $>1.5$ years (>80 weeks). In 2009, no activity was detected, indicating that the source remained in quiescence. However, in 2010 June the source is again detected in outburst by Swift/XRT at a $2-10 \mathrm{keV}$ luminosity of a few times $10^{35} \mathrm{erg} \mathrm{s}^{-1}$ (Degenaar et al. 2010).

The different outburst spectra of AX J1745.6-2901 are displayed in Fig. 3. Both the 2006 and 2007-2008 outburst have a soft X-ray spectrum with powerlaw indices of $\Gamma=2.4 \pm 0.1$ and $2.7 \pm 0.1$, respectively (see Table 1 ). The 2006 outburst appears to have a harder X-ray spectrum than the brighter 2007-2008 outburst (we obtain similar results when $N_{\mathrm{H}}$ is left as a free parameter and not fixed between the outbursts). The spectra indicate that the system is heavily absorbed with a best fit hydrogen column density of $N_{\mathrm{H}}=(23.8 \pm 0.5) \times 10^{22} \mathrm{~cm}^{-2}$. The average 2-10 keV unabsorbed flux during the 2007-2008 outburst was $\sim 1 \times 10^{-10} \mathrm{erg} \mathrm{cm}^{-2} \mathrm{~s}^{-1}$. For an outburst duration of $>80$ weeks, this implies a fluence of $\gtrsim 7 \times 10^{-3} \mathrm{erg} \mathrm{cm}^{-2}$, which is a factor of $\sim 10$ higher than that of the 2006 outburst (see Table 2).

\subsection{CXOGC J174535.5-290124}

The X-ray source CXOGC J174535.5-290124 is located only $\sim 14^{\prime \prime}$ away from AX J1745.6-2901 (see Fig. 1). This transient was discovered in 2001 during a monitoring campaign of the GC with Chandra (Muno et al. 2004). Since then, the source has been detected in outburst multiple times with Chandra, XMM-Newton and Swift, displaying typical luminosities of $\sim 10^{33-34} \mathrm{erg} \mathrm{s}^{-1}$ (Muno et al. 2005; Wijnands et al. 2005, 2006b; Degenaar et al. 2008a; Degenaar \& Wijnands 2009). In quiescence, the source has not been detected, yielding an upper limit on luminosity of $<9 \times 10^{30} \mathrm{erg} \mathrm{s}^{-1}$ (2-8 keV, Muno et al. 2005).

Swift/XRT cannot spatially resolve CXOGC J174535.5290124 and AX J1745.6-2901 when the latter, which is the brightest of the two, is active. We can therefore only deduce information on the activity of CXOGC J174535.5-290124 from Swift data obtained in epochs that AX J1745.6-2901 is quiescent, which is 2006 July-November and 2008 September onwards (see Sect. 3.1). In 2006, the Swift/XRT observations captured an outburst from CXOGC J174535.5-290124 that had a duration of $>12$ weeks (Degenaar \& Wijnands 2009).

Renewed activity of CXOGC J174535.5-290124 was revealed by Chandra observations obtained on 2008 May 1011, when the source displayed a 2-10 keV luminosity of a few times $10^{33} \mathrm{erg} \mathrm{s}^{-1}$ (Degenaar et al. 2008b). After AX J1745.6-2901 had returned to quiescence in 2008 September, CXOGC J174535.5-290124 was continuously detected until the monitoring observations ended on 2008 October 30 . This outburst thus lasted for $>8$ weeks. If the activity observed by Swift/XRT was part of the same outburst that was detected by Chandra in 2008 May, the outburst duration increases to $>24$ weeks. Alternatively, if the source returned to quiescence in between, the dormant phase must have lasted $<18$ weeks. The source was not found active during the 2009 observations (see Fig. 2).

The average $2-10 \mathrm{keV}$ unabsorbed flux observed with Swift/XRT in 2008 was $\sim 1 \times 10^{-12} \mathrm{erg} \mathrm{cm}^{-2} \mathrm{~s}^{-1}$, a factor $\sim 1.5$ lower than observed in 2006 (see Table 1). For an outburst duration of $>8$ weeks, we can constrain the fluence of the 2008 outburst to be $>7 \times 10^{-6} \mathrm{erg} \mathrm{cm}^{-2}(2-10 \mathrm{keV})$. This increases to $>2 \times 10^{-5} \mathrm{erg} \mathrm{cm}^{-2}$ if the outburst endured for more than 24 weeks. The spectrum of CXOGC J174535.5-290124 is heavily absorbed $\left(N_{\mathrm{H}}=(12.0 \pm 6.9) \times 10^{22} \mathrm{~cm}^{-2}\right)$ and for both outbursts we obtain a rather hard spectral index of $\Gamma \sim 1$, although the uncertainties on this parameter are very large (see Table 1). The X-ray spectra of the 2006 and 2008 outbursts are shown in Fig. 3.

\subsection{GRS 1741-2853}

GRS 1741-2853 was discovered in 1990 March-April by the Granat satellite (Sunyaev 1990). Since then, the system has been detected in outburst on multiple occasions with $2-10 \mathrm{keV}$ luminosities of $\sim 10^{36} \mathrm{erg} \mathrm{s}^{-1}$ (e.g., Sakano et al. 2002; Muno et al. 2003a; Wijnands et al. 2006a; Trap et al. 2009). In quiescence the source displays a luminosity of $\sim 10^{32} \mathrm{erg} \mathrm{s}^{-1}$ (Muno et al. 2003a). The detection of thermonuclear X-ray bursts by BeppoSAX established that this system is an LMXB harboring a neutron star (Cocchi et al. 1999).

Between 2006 September 14-20, the Swift/XRT monitoring observations detected a short $(\sim 1$ week) outburst from GRS 1741-2853, which reached a $2-10 \mathrm{keV}$ peak luminosity of only $\sim 9 \times 10^{34} \mathrm{erg} \mathrm{s}^{-1}$. This is significantly lower than other outbursts observed from this source $\left(L_{\mathrm{X}} \sim 10^{36-37} \mathrm{erg} \mathrm{s}^{-1}\right)$. In 2007 February-April, the source experienced a longer ( $>13$ weeks) and brighter (2-10 keV peak luminosity of $\sim 2 \times$ $10^{36} \mathrm{erg} \mathrm{s}^{-1}$ ) accretion outburst, which was captured by several satellites (Kuulkers et al. 2007b; Porquet et al. 2007; Muno et al. 2007; Degenaar \& Wijnands 2009).

GRS 1741-2853 remained dormant throughout the 2008 Swift monitoring campaign (see Fig. 2), but experienced another accretion outburst in 2009 October, which was registered by Integral and Swift (Chenevez et al. 2009; Kennea 2009). While GRS 1741-2853 was not detected during Swift/XRT observations performed on 2009 September 23, it was found active on 2009 September 29 and the flux started to rise in the following days (Kennea 2009; see also Fig. 2). The source intensity had decayed to the background level during the last observation of the campaign, performed on 2009 November 1. This suggests that the 2009 outburst had a duration of $4-5$ weeks. The outburst reached a peak luminosity of $\sim 1 \times 10^{37} \mathrm{erg} \mathrm{s}^{-1}$, while the average outburst value was $\sim 2 \times 10^{36} \mathrm{erg} \mathrm{s}^{-1}(2-10 \mathrm{keV}$; see Table 1). To our knowledge, this is the highest peak luminosity ever reported for GRS 1741-2853.

By fitting the data of the three outbursts simultaneously, we obtain a hydrogen column density of $N_{\mathrm{H}}=(14.0 \pm 0.7) \times$ $10^{22} \mathrm{~cm}^{-2}$. The X-ray spectra, displayed in Fig. 3, are soft with powerlaw indices of $\Gamma=5.0 \pm 2.5,2.6 \pm 0.1$ and $3.0 \pm 0.2$ for the 2006, 2007 and 2009 activity, respectively. We note that our values for both $N_{\mathrm{H}}$ and $\Gamma$ are a bit higher than obtained from Chandra and XMM-Newton observations, albeit largely consistent within the errors (Muno et al. 2003a; Wijnands et al. 2006a; Trap et al. 2009). The uncertainty on the spectral index 
of the short and weak 2006 outburst is very large, but comparing the 2007 and 2009 outburst data suggests that the X-ray spectrum is softer for the brightest of the two outbursts. Using a duration of 4-5 weeks and an average unabsorbed flux of $\sim 2.8 \times 10^{-10} \mathrm{erg} \mathrm{cm}^{-2} \mathrm{~s}^{-1}$ (see Table 1), we can estimate that the 2009 outburst of GRS $1741-2853$ had a $2-10 \mathrm{keV}$ fluence of $\sim 8 \times 10^{-4} \mathrm{erg} \mathrm{cm}^{-2}$. Despite the different outburst duration and peak luminosity, this is comparable to the 2007 outburst observed by Swift/XRT (see Table 2).

\subsection{XMM J174457-2850.3}

XMM J174457-2850.3 is a transient X-ray source that was first detected in outburst with XMM-Newton in 2001 September, when it displayed a 2-10 keV luminosity of $\sim 5 \times 10^{34} \mathrm{erg} \mathrm{s}^{-1}$ (Sakano et al. 2005). Since its initial discovery, XMM J1744572850.3 has been active repeatedly, displaying 2-10 keV X-ray luminosities in a broad range of a few times $\sim 10^{33} \mathrm{erg} \mathrm{s}^{-1}$, up to $\sim 10^{36} \mathrm{erg} \mathrm{s}^{-1}$ (Wijnands et al. 2006a; Muno et al. 2007).

As mentioned earlier, XMM J174457-2850.3 is only in FOV in a sub-set of the Swift/XRT monitoring data, due to its relatively large offset from Sgr A* $\left(\sim 13.7^{\prime}\right)$. The source was never in FOV during the 2006 observations. In 2007, the source field was covered a few times between July and November, and XMM J174457-2850.3 was detected at 2-10 keV luminosities of $\sim 10^{33-34} \mathrm{erg} \mathrm{s}^{-1}$ (Degenaar \& Wijnands 2009). When the source first came into view in 2008 , on June 28, it displayed a $2-10 \mathrm{keV} X$-ray luminosity of $\sim 1 \times 10^{36} \mathrm{erg} \mathrm{s}^{-1}$. The source intensity decreased over the course of a few days, down to a level of a few times $10^{33} \mathrm{erg} \mathrm{s}^{-1}$ around 2008 July 7 (see Fig. 2).

Following this decay, the source remained to be detected by Swift/XRT all through the end of the monitoring observations on 2008 October 30. During this episode, XMM J174457-2850.3 displayed a 2-10 keV luminosity of a few times $\sim 10^{33} \mathrm{erg} \mathrm{s}^{-1}$, which is a factor $>10$ above its quiescent level of $\sim 10^{32} \mathrm{erg} \mathrm{s}^{-1}$ (Sakano et al. 2005). We extracted separate spectra of the bright outburst (2008 June 28-July 7), as well as the low-level activity that followed (2008 July 8-October 30 ). Both spectra are shown in Fig. 3 and the spectral parameters and fluxes are listed in Table 1. We note that the source is not detected in our Chandra monitoring observations of the GC performed on 2008 May 10 (Degenaar et al., in preparation), which implies that the 2$10 \mathrm{keV}$ luminosity of XMM J174457-2850.3 was lower than a few times $10^{33} \mathrm{erg} \mathrm{s}^{-1}$ at that time. The bright active state (2$10 \mathrm{keV}$ luminosity of $\sim 10^{35-36} \mathrm{erg} \mathrm{s}^{-1}$ ) detected in 2008 lateJune thus lasted $<49$ days ( $<7$ weeks).

In 2009, the source was detected during a single Swift pointing performed on September 29, at a luminosity of $\sim 2 \times$ $10^{35} \mathrm{erg} \mathrm{s}^{-1}(2-10 \mathrm{keV})$. The spectrum of this observation largely overlays the average spectrum of the 2008 outburst and is therefore not plotted in Fig. 3. XMM J174457-2850.3 is not active in the preceding, nor in the subsequent observation, carried out on September 23 and October 2, respectively. This implies that the activity lasted less than 9 days.

For the different outbursts captured by Swift between 2006-2009, we obtain spectral parameters that are comparable to the outburst values reported by Sakano et al. (2005) using XMM-Newton and Chandra data obtained in 2001. The source is heavily absorbed $\left(N_{\mathrm{H}}=(7.5 \pm 2.9) \times 10^{22} \mathrm{~cm}^{-2}\right)$ and the powerlaw index adapts values of $\Gamma \sim 1.5-2.5$, with large uncertainties due to the low statistics (see Table 1). There is no obvious correlation between the spectral index and the source flux. The $2-10 \mathrm{keV}$ fluence of the different outbursts of
XMM J174457-2850.3 varies between $\sim(0.4-10) \times$ $10^{-5} \mathrm{erg} \mathrm{cm}^{-2}$ (see Table 2), with an average value of $\sim 2.5 \times$ $10^{-5} \mathrm{erg} \mathrm{cm}^{-2}$.

\subsection{CXOGC J174538.0-290022}

The X-ray source CXOGC J174538.0-290022 was discovered during Chandra monitoring observations of the GC (Muno et al. 2003b). Between 1999 and 2004, Chandra detected the source at a minimum and maximum luminosity of $\sim 1 \times 10^{33}$ and $\sim 3 \times 10^{34} \mathrm{erg} \mathrm{s}^{-1}$, respectively (2-8 keV, Muno et al. 2005). The source was not detected during the Swift/XRT monitoring observations carried out in 2006, 2007 and 2008 (see Fig. 2).

CXOGC J174538.0-290022 was reported active as seen during XMM-Newton observations obtained between 2009 April 15 , displaying a 2-10 keV luminosity of $\sim 2 \times 10^{34} \mathrm{erg} \mathrm{s}^{-1}$ (Ponti et al. 2009). A $4.6 \mathrm{ks}$ Swift/XRT ToO pointing performed on 2009 May 17, about 6 weeks after the XMM-Newton detection, found the source still in outburst. The Swift monitoring observations of the GC resumed on 2009 June 4, and the source is clearly detected by visual inspection until 2009 mid-July. The average luminosity during this episode is $\sim 9 \times 10^{34} \mathrm{erg} \mathrm{s}^{-1}$, peaking at $\sim 2 \times 10^{35} \mathrm{erg} \mathrm{s}^{-1}(2-10 \mathrm{keV})$.

Although no clear source activity is apparent in individual exposures obtained after 2009 mid-July, summing the data from this period till the end of the monitoring observations (2009 November 1), does result in a weak detection of the source. The average luminosity during this episode is $\sim 1 \times 10^{34} \mathrm{erg} \mathrm{s}^{-1}$. Figure 3 displays the average Swift/XRT spectrum of the entire active period, i.e., from 2009 May-November. The spectral parameters and fluxes are listed in Table 1.

The outburst captured by Swift had a duration of $>9$ weeks. It is likely that the source was continuously active between the $X M M$-Newton detection and the Swift/XRT observations, which would imply that the outburst had a duration of $>30$ weeks. We note that the source is not detected during the 2010 monitoring observations, which commenced on 2010 April 4. This implies that the outburst of CXOGC J174538.0-290022 was shorter than 52 weeks. The spectral parameters deduced from fitting the XRT data $\left(N_{\mathrm{H}}=(12.8 \pm 5.9) \times 10^{22} \mathrm{~cm}^{-2}\right.$ and $\Gamma=1.4 \pm 0.9$; see Table 1) are similar to the values inferred from XMM-Newton observations obtained in 2008 early-April (Ponti et al. 2009). If we assume an outburst duration of 30-52 weeks, the average unabsorbed flux of $\sim 5 \times 10^{-12} \mathrm{erg} \mathrm{cm}^{-2} \mathrm{~s}^{-1}$ implies a $2-10 \mathrm{keV}$ outburst fluence of $\sim(9-20) \times 10^{-5} \mathrm{erg} \mathrm{cm}^{-2}$ (see Table 2).

\section{Discussion}

In this work, we analyzed the lightcurves and spectra of five different X-ray transients that were found active during Swift/XRT monitoring observations of the GC carried out in 2008 and 2009. The four sources AX J1745.62901, CXOGC J174535.5-290124, GRS 1741-2853 and XMM J174457-2850.3 were also active between 2006 and 2007, while CXOGC J174538.0-290022 was detected for the first time with Swift in 2009. The two brightest transients, AX J1745.6-2901 and GRS 1741-2853, are both confirmed neutron star LMXBs (based on the detection of thermonuclear X-ray bursts), while the other three are unclassified X-ray sources.

AX J1745.6-2901 was observed to return to quiescence in 2008 September, following an unusually long accretion episode that started before 2007 February and endured for $\gtrsim 1.5$ years. In 2009, the Swift/XRT observations captured the brightest outburst ever reported for GRS 1741-2853, which reached up to a 
$2-10 \mathrm{keV}$ peak luminosity of $1.3 \times 10^{37} \mathrm{erg} \mathrm{s}^{-1}$. Both sources appear to have rather soft X-ray spectra with powerlaw indices that are higher $(\Gamma \sim 2.5-3.0$; see Table 1$)$ than typically found for brighter neutron star LMXBs $(\Gamma \sim 2)$. Furthermore, the 20082009 data set reveals that although XMM J174457-2850.3 exhibits outbursts with peak luminosities around $\sim 1 \times 10^{36} \mathrm{erg} \mathrm{s}^{-1}$, it can also spend long episodes at a much lower active level of $\sim 10^{33-34} \mathrm{erg} \mathrm{s}^{-1}(2-10 \mathrm{keV})$.

CXOGC J174535.5-290124 and CXOGC J174538.0290022 both display very low $2-10 \mathrm{keV}$ peak luminosities of $\sim 10^{34-35} \mathrm{erg} \mathrm{s}^{-1}$ and have never been detected at higher levels. CXOGC J174535.5-290124 was active in 2008, while a previous outburst was detected with Swift/XRT in 2006 (Degenaar \& Wijnands 2009). This confirms that this system has a relatively high duty cycle. CXOGC J174538.0-290022 displayed an outburst peak luminosity of $\sim 2 \times 10^{35} \mathrm{erg} \mathrm{s}^{-1}(2-10 \mathrm{keV})$, which is $\sim 2$ orders of magnitude higher than the lowest luminosity detected during Chandra observations of the GC carried out between 1999 and $2004\left(\sim 1 \times 10^{33} \mathrm{erg} \mathrm{s}^{-1}\right.$ in the $2-8 \mathrm{keV}$ band, Muno et al. 2005). This unambiguously demonstrates the transient nature of this source. If CXOGC J174538.0-290022 is an $\mathrm{X}$-ray binary and its quiescent luminosity is $\sim 1 \times 10^{33} \mathrm{erg} \mathrm{s}^{-1}$ (Muno et al. 2005), this would favor a neutron star as the compact primary, since black hole systems are typically fainter in their quiescent states unless the orbital period is several days (e.g., Narayan et al. 1997; Menou et al. 1999; Lasota 2007).

In 2006, the Swift/XRT monitoring campaign of the GC detected activity of three other transients, CXOGC J174540.0290005, Swift J174553.7-290347 (likely associated to CXOGC J174553.8-290346) and Swift J174622.1-290634 (Degenaar \& Wijnands 2009). The former two both experienced short outbursts ( $\sim 2$ weeks) with $2-10 \mathrm{keV}$ peak luminosities of $\sim 2 \times 10^{35} \mathrm{erg} \mathrm{s}^{-1}$. These two sources are not detected during the 2008-2009 observations, which confirms that these systems have low duty cycles (Degenaar \& Wijnands 2009; see also Table 2). The newly discovered transient Swift J174622.1290634 was active for $\sim 5$ weeks in 2006 and reached a peak luminosity of $\sim 7 \times 10^{34} \mathrm{erg} \mathrm{s}^{-1}(2-10 \mathrm{keV})$. This source has a relatively large offset from Sgr $\mathrm{A}^{*}\left(\sim 11^{\prime}\right)$ and was only in FOV during 39 and 4 pointings in 2008 and 2009, respectively. No activity is detected from the source during these observations.

\subsection{Peculiar source properties}

\subsubsection{Lightcurve morphology of AX J1745.6-2901}

As discussed in Sect. 3.1, the Swift/XRT observations of the GC exposed two distinct outbursts from AX J1745.6-2901 between 2006 and 2009, which are very different in terms of duration and luminosity (see Tables 1 and 2). Since AX J1745.6-2901 is a confirmed neutron star LMXB, the disk instability model is thought to provide the framework to explain the outburst behavior of this source.

The average $2-10 \mathrm{keV}$ luminosity during the 2007-2008 outburst was $\sim 1 \times 10^{36} \mathrm{erg} \mathrm{s}^{-1}$ (see Table 1). Assuming that the bolometric luminosity is a factor of $\sim 3$ higher (e.g., in 't Zand et al. 2007), this implies a mass-accretion rate of $\langle\dot{M}\rangle_{\mathrm{ob}} \sim$ $3 \times 10^{-10} M_{\odot} \mathrm{yr}^{-1}$ for a canonical neutron star with $M=1.4 M_{\odot}$ and $R=10 \mathrm{~km}$. For an outburst duration of 1.5 years, this corresponds to a total accreted disk mass of $\sim 5 \times 10^{-10} M_{\odot}$. In 2006 , the outburst had an average luminosity of $\sim 4 \times 10^{35} \mathrm{erg} \mathrm{s}^{-1}$ and a duration of $>16$ weeks, which would translate into a mean massaccretion rate of $\langle\dot{M}\rangle_{\mathrm{ob}} \sim 1 \times 10^{-10} M_{\odot} \mathrm{yr}^{-1}$ and a total accreted mass of $\gtrsim 3 \times 10^{-11} M_{\odot}$.
Given the fact that AX J1745.6-2901 is transient, the masstransfer rate from the companion star must be lower than the accretion rate onto the compact object during outburst (e.g., King \& Ritter 1998), i.e., $\langle\dot{M}\rangle_{\mathrm{tr}} \lesssim 1 \times 10^{-10} M_{\odot} \mathrm{yr}^{-1}$ (as estimated from the 2006 outburst; see Table 2). If the mass-transfer rate from the companion star does not change considerably over time, it would thus take the system at least 5 years to build up the accretion disk that powered the 2007-2008 outburst from scratch. This is consistent with the fact that no similarly long outbursts from this source have been observed between 1994 and 2006 (see Sect. 3.1). The duty cycle of similar 1.5-year long outbursts from this system would thus be $\$ 23 \%$. This is in agreement with observational constraints, which result in an estimated duty cycle of 10-30\% (Degenaar \& Wijnands 2009). Given the time required to build up an accretion disk that can account for the 2007-2008 activity, and the observed quiescence interval between the 2006 and 2007-2008 outbursts of only 4-7 months (Degenaar \& Wijnands 2009; see also Fig. 2), it seems that a significant residual accretion disk must have remained after the 2006 outburst ended. Shorter outbursts like the one observed in 2006 consume much less disk mass and could recur on a timescale of a only a few months. As mentioned in Sect. 3.1, AX J1745.6-2901 was again reported in outburst in 2010 June, displaying a similar intensity level as in 2006 (Degenaar et al. 2010).

Within the disk instability model, we can understand the observed behavior if in 2006 only part of the accretion disk became ionized, while the 2007-2008 outburst drained a larger part of (or maybe the full) accretion disk (see, e.g., King \& Ritter 1998; Lasota 2001). This picture might also provide an explanation for the fact that the 2006 outburst was fainter than the one observed in 2007-2008, since the mass-accretion rate (and thus the accretion luminosity) is expected to scale with the size of the hot ionized zone of the accretion disk (see King \& Ritter 1998). We note that the disk instability model for accreting white dwarfs predicts alternating sequences of outbursts with different duration and brightness, consistent with observations of dwarf novae eruptions (e.g., Cannizzo 1993; Lasota 2001). While driven by the same underlying mechanism, it is thought that in LMXBs the stability properties are strongly influenced by irradiation of the accretion disk (King \& Ritter 1998; Lasota 2001). As a consequence, LMXBs are expected to consume a larger part of the accretion disk during outbursts, which are therefore longer and less frequent than observed for dwarf novae (King \& Ritter 1998; Lasota 2001).

\subsubsection{Recurrence time of GRS 1741-2853}

Despite the different duration and average flux, the fluence of the 2009 outburst of GRS 1741-2853 is comparable to the 2007 outburst fluence of $\sim 1 \times 10^{-3} \mathrm{erg} \mathrm{cm}^{-2}$ (see Table 2). The two outbursts are separated by an epoch of $\sim 2.5$ year. The total mass accreted during the 2007 outburst can be estimated as $\gtrsim 7 \times 10^{-11} M_{\odot}$ (for $t_{\mathrm{ob}}>13$ weeks and $\langle\dot{M}\rangle_{\mathrm{ob}} \sim$ $3 \times 10^{-10} M_{\odot} \mathrm{yr}^{-1}$ ). For the 2009 outburst we obtain a comparable value of $\sim 4 \times 10^{-11} M_{\odot}\left(t_{\mathrm{ob}} \sim 4-5\right.$ weeks and $\langle\dot{M}\rangle_{\mathrm{ob}} \sim$ $\left.5 \times 10^{-10} M_{\odot} \mathrm{yr}^{-1}\right)$.

In 2005, GRS 1741-2853 also underwent an accretion outburst that endured for several weeks. The rise of this outburst was captured by Integral in 2005 early-April (Kuulkers et al. 2007c), while Chandra observations indicated that the source was fading in 2005 early-July (Wijnands et al. 2006a). This suggests an outburst duration of $\sim 13$ weeks. Assuming an average 2-10 keV flux of $\sim 1 \times 10^{-10} \mathrm{erg} \mathrm{cm}^{-2} \mathrm{~s}^{-1}$ (as inferred from 
Chandra observations performed in 2005 June, Wijnands et al. 2006a), we can asses that the 2005 outburst had a $2-10 \mathrm{keV}$ fluence of approximately $\sim 8 \times 10^{-4} \mathrm{erg} \mathrm{cm}^{-2}$. This is comparable to the two large outbursts occurring in 2007 and 2009 (see Table 2). The time between the 2005 and the 2007 outburst is nearly 2 years. The total mass accreted during the 2005 outburst can be estimated as $\sim 5 \times 10^{-11} M_{\odot}$ (assuming $t_{\mathrm{ob}} \sim 13$ weeks and $\left.\langle\dot{M}\rangle_{\mathrm{ob}} \sim 2 \times 10^{-10} M_{\odot} \mathrm{yr}^{-1}\right)$. This is very similar to the values estimated above for the 2007 and 2009 outbursts.

Based on the three outbursts observed for GRS 1741-2853 in the past 5 years $(2005,2007$ and 2009, neglecting the weak and short outburst captured by Swift in 2006) we can infer that the system has typical outburst duration on the order of $\sim 10$ weeks. The detection history in the past decade (see Trap et al. 2009, for an overview), suggests a recurrence time of roughly $\sim 2$ years. This implies a duty cycle of $\sim 10 \%$ and the average accretion rate during outburst appears to be typically a few times $10^{-10} M_{\odot} \mathrm{yr}^{-1}$.

\subsubsection{XMM J174457-2850.3: a wind-fed system?}

As discussed in Section 3.4, the unclassified transient X-ray source XMM J174457-2850.3 has a quiescent level of $L_{\mathrm{X}} \sim$ $10^{32} \mathrm{erg} \mathrm{s}^{-1}$, while the observed maximum luminosity is $\sim 10^{36} \mathrm{erg} \mathrm{s}^{-1}$ (2-10 keV). The 2008 Swift/XRT observations of the GC show that the bright stages of this source might only last for a few days, while XMM J174457-2850.3 is often found at levels intermediate between quiescence and full outburst, at a 2$10 \mathrm{keV}$ luminosity of $\sim 10^{33-34} \mathrm{erg} \mathrm{s}^{-1}$. Such behavior is difficult to understand within the framework of accretion disk instabilities in LMXBs. Instead, wind accretion might provide a more natural explanation.

The activity displayed by XMM J174457-2850.3 is in some ways reminiscent of the behavior observed from SFXTs, which harbor neutron stars accreting from the stellar wind of a supergiant O/B companion (e.g., Negueruela et al. 2006). These systems undergo sporadic X-ray flares lasting only a few hours to days and reaching up to $2-10 \mathrm{keV}$ luminosities of $\sim 10^{36-37} \mathrm{erg} \mathrm{s}^{-1}$ (e.g., Sidoli 2009). They seem to reside in their quiescent states $\left(L_{X} \sim 10^{32} \mathrm{erg} \mathrm{s}^{-1}\right)$ only occasionally, and instead linger the majority of their time at levels of $\sim 10^{33-34} \mathrm{erg} \mathrm{s}^{-1}$ displaying X-ray spectra that are well fit by a powerlaw model with a photon index in the range 1-2 (2$10 \mathrm{keV}$, e.g., Sidoli et al. 2008). Slow (i.e., a few seconds to minutes) pulsations have been detected from a few of these systems (e.g., Sidoli 2009). An HMXB configuration would be consistent with the possible detection of $5.25 \mathrm{~s}(0.19 \mathrm{~Hz})$ pulsations from XMM J174457-2850.3 in XMM-Newton observations (Sakano et al. 2005). However, since the data analysis was limited by both statistics and exposure, the reliability of the coherent signal was considered highly uncertain by these authors and this result therefore needs to be verified.

Laycock et al. (2005) report on I-band images of the field around XMM J174457-2850.3, obtained during an X-ray outburst in 2005 early-June. Using the IMACS imaging spectrograph mounted at the Magellan-Baade telescope, these authors detect an optical source with $I=22.04 \pm 0.1 \mathrm{mag}$ and $R-I=$ $1.95 \pm 0.2$, a low extinction $\left(A_{\mathrm{V}} \sim 7 \mathrm{mag}\right)$ and no variability. However, this object is located $\sim 3^{\prime \prime} \mathrm{NE}$ from the Chandra coordinates of XMM J174457-2850.3, lying outside the 1.5" positional uncertainty (Wijnands et al. 2006a), and is therefore likely not related. This implies that the optical counterpart of XMM J174457-2850.3 has a magnitude $I>25.6$ mag ( $3 \sigma$ upper limit, Laycock et al. 2005). Finding an optical counterpart is hampered by the large extinction in the direction of the source. Using the relation of Predehl \& Schmitt (1995), a hydrogen column of $N_{\mathrm{H}} \sim 7.5 \times 10^{22} \mathrm{~cm}^{-2}$ (as inferred from fitting X-ray spectral data; see Sect. 3.4) would translate into a visual extinction of $A_{\mathrm{V}} \sim 42 \mathrm{mag}$. Since the extinction is much lower at longer wavelengths, it might be more fruitful to search for a counterpart in the infrared.

A recent study by Mauerhan et al. (2009) did not reveal any infrared objects associated with XMM J174457-2850.3, up to a limiting magnitude of $K_{\mathrm{s}} \lesssim 15.6 \mathrm{mag}$. The extinction in the $K_{\mathrm{s}}$ band can be estimated as $A_{K \mathrm{~s}}=0.062 \times A_{\mathrm{V}} \sim$ 2.6 mag (Nishiyama et al. 2008). Using the tables of Drilling \& Landolt (2000) and Tokunaga (2000) suggests that the survey of Mauerhan et al. (2009) should have enabled the detection of an O/B supergiant $\left(K_{\mathrm{s}} \sim 11 \mathrm{mag}\right)$, as well as a main sequence star with spectral type earlier than B3V. Since most known HMXBs have donor stars with spectral types from O9VB2V (Negueruela 1998), XMM J174457-2850.3 is not likely to be an HMXB unless the source is more distant than $8 \mathrm{kpc}$. Its behavior therefore remains puzzling.

Another possibility to explore is whether this source could be an LMXB in which a neutron star is accreting from the wind of an M-gaint companion. Currently, only 8 of such symbiotic X-ray binaries have been identified (e.g., Masetti et al. 2007; Nespoli et al. 2010). All of these systems show both long- and short-term X-ray variability and are characterized by $2-10 \mathrm{keV}$ luminosities ranging between $\sim 10^{32-35} \mathrm{erg} \mathrm{s}^{-1}$, although one object shows more intense X-ray emission of $L_{\mathrm{X}} \sim 10^{36-37} \mathrm{erg} \mathrm{s}^{-1}$ (e.g., Masetti et al. 2007). However, for the extinction towards XMM J174457-2850.3 and a distance of $8 \mathrm{kpc}$, an M-type giant would have a magnitude of $K_{\mathrm{s}} \sim 11-13 \mathrm{mag}$ (Drilling \& Landolt 2000; Tokunaga 2000). The lack of a counterpart with $K_{\text {s }} \lesssim 15.6$ mag (Mauerhan et al. 2009) therefore renders this scenario unlikely as well unless the source is located at a larger distance than $8 \mathrm{kpc}$.

\subsection{Mass-accretion rates}

Amongst the different transients that are detected during the Swift/XRT monitoring observations of the GC there are two LMXBs (AX J1745.6-2901 and GRS 1741-2853), whereas the others remain unclassified. However, the energies involved in their outburst phenomena make it likely that these harbor accreting neutron stars or black holes. Recently, Mauerhan et al. (2009) searched for near-infrared counterparts to X-ray sources located towards the GC. Their catalog reveals no counterparts for any of the unclassified transients detected during the Swift campaign, with a limiting magnitude of $K_{\mathrm{s}} \lesssim 15.6 \mathrm{mag}$. This suggests that these systems are likely transient LMXBs rather than HMXBs (Muno et al. 2005; Mauerhan et al. 2009). It is therefore interesting to estimate the mean accretion rate during the outbursts of these systems, $\langle\dot{M}\rangle_{\text {ob }}$, from which we can obtain an order of magnitude approximation for the long-term averaged mass-accretion rates, when combined with estimates of their duty cycles.

The time-averaged mass-accretion rate, $\langle\dot{M}\rangle_{\text {long }}$, is an important parameter for binary evolution models that attempt to explain the nature of low-luminosity LMXBs (e.g., King \& Wijnands 2006). We refer to Degenaar \& Wijnands (2009) for the details of such calculations and the associated caveats. Here, we only calculate the (time-averaged) mass-accretion rates assuming a neutron star primary with $M_{\mathrm{NS}}=1.4 M_{\odot}$ and $R_{\mathrm{NS}}=$ $10 \mathrm{~km}$. These results are listed in Table 2 . In case of a black hole accretor with $M_{\mathrm{BH}}=10 M_{\odot}$ and $R_{\mathrm{BH}}=30 \mathrm{~km}$, the values 
given in this table can be multiplied with a factor $\sim 0.4$, although one should bear in mind the caveats discussed in Degenaar \& Wijnands (2009).

The duty cycles of AX J1745.6-2901 and GRS 1741-2853 have been estimated in Sects. 4.1.1 and 4.1.2, respectively. For a discussion on the outburst and quiescent timescales of CXOGC J174535.5-290124 and XMM J174457-2850.3 we refer to Degenaar \& Wijnands (2009), since the new 2008-2009 data leaves those estimates unaltered. As mentioned in Sect. 4, the Swift monitoring observations detected activity of three transients in 2006 (Degenaar \& Wijnands 2009), that did not recur in 2008-2009. Since Swift J174622.1-290634 was in FOV only during a small number of pointings, we cannot refine the time-averaged mass-accretion rate for this source. However, for CXOGC J174540.0-290005 and Swift J174553.7-290347, we can put further constraints on the time that these systems spend in quiescence. In 2008, daily observations were carried out for 36 consecutive weeks, which can thus be used as a lower limit on the quiescent timescale of the two transients. Both CXOGC J174540.0-290005 and Swift J174553.7-290347 exhibited an outburst with a duration of two weeks in 2006. This new constraint then puts their duty cycles at $\lesssim 5 \%$. Based on the 2006-2007 data set, duty cycles of $\$ 6 \%$ and $\lesssim 8 \%$ were estimated for CXOGC J174540.0-290005 and Swift J174553.7290347, respectively (Degenaar \& Wijnands 2009). Since the former was also active in 2003 (Muno et al. 2005), the lower limit on its duty cycle is $\gtrsim 1 \%$ (Degenaar \& Wijnands 2009).

CXOGC J174538.0-290022 was detected for the first time during the Swift/XRT observations performed in 2009. The source was observed with a peak outburst luminosity of $\sim 2 \times$ $10^{35} \mathrm{erg} \mathrm{s}^{-1}$. Apart from the 2009 activity, CXOGC J174538.0290022 has been detected with a luminosity exceeding $1 \times$ $10^{34} \mathrm{erg} \mathrm{s}^{-1}$ only once before, in 1999 September with Chandra (Muno et al. 2005). This implies that the quiescent timescale of this source is less than 10 years. On the other hand, the source was not found active throughout the 2006-2008 Swift/XRT monitoring campaign. During those years, nearly daily observations were carried out, only interrupted for 17 weeks between 2006 November and 2007 March, for 15 weeks in the epoch 2007 November-2008 February (both due to Sun-angle constraints), and for 6 weeks between 2007 August 11-September 26 (due to a safe-hold event, Gehrels 2007). If an outburst duration of $30-52$ weeks is typical for this source, we can thus put a lower limit on the quiescent timescale of $\sim 2.7$ years (139 weeks), although shorter outbursts might have been missed. The duty cycle of this source is then roughly between $\sim 5-20 \%$, which results in a time-averaged mass-accretion rate of $\langle\dot{M}\rangle_{\text {long }} \sim(5-20) \times$ $10^{-13} M_{\odot} \mathrm{yr}^{-1}$ (see Table 2). Despite the apparent long outburst duration, the estimated time-averaged accretion rate is amongst the lowest of the transients detected in the Swift/XRT monitoring campaign of the GC.

It can be seen from Table 2 that the two confirmed neutron star LMXBs AX J1745.6-2901 and GRS 1741-2853 have estimated time-averaged mass-accretion rates of a few times $10^{-11} M_{\odot} \mathrm{yr}^{-1}$, which is not extraordinary low compared to other LMXBs. XMM J174457-2850.3 is also amongst the brightest transients detected during the Swift monitoring observations $\left(L_{X, \text { peak }} \sim 10^{36} \mathrm{erg} \mathrm{s}^{-1}\right)$ and this system appears to recur quite often. This results in a relatively high time-averaged massaccretion rate $\left(10^{-12}-10^{-11} M_{\odot} \mathrm{yr}^{-1}\right)$ compared to the other five transients listed in Table 2, which have lower outburst luminosities and lower estimated rates of $\langle\dot{M}\rangle_{\text {long }} \lesssim 2 \times 10^{-12} M_{\odot} \mathrm{yr}^{-1}$.

As mentioned above, the time-averaged mass-accretion rate is an important parameter for binary evolution models.
King \& Wijnands (2006) construct a theoretical toy model exploring the evolution of LMXBs at low accretion luminosities. The estimates of these authors show that if objects like CXOGC J174535.5-290124, CXOGC J174538.0290022, CXOGC J174540.0-290005, Swift J174553.7-290347 and Swift J174622.1-290634 are indeed X-ray binaries, their time-averaged mass-accretion rates suggests that the massdonors are likely very low-mass or hydrogen-depleted stars. However, further refinement of their duty cycles and outburst energetics, as well as detailed evolutionary calculations, are required to grasp the nature of these peculiar objects.

\subsection{Summary of the campaign 2006-2009}

Starting in 2006 February and extending into 2009, the Swift/XRT monitoring campaign of the GC detected activity of 8 different transients in total, from which 14 distinct outbursts were observed. All sources have experienced outbursts with peak 2-10 keV luminosities $L_{\mathrm{X} \text {, peak }} \lesssim 10^{36} \mathrm{erg} \mathrm{s}^{-1}$, although the two neutron star LMXBs AX J1745.6-2901 and GRS 17412853 both displayed brighter outbursts as well $\left(L_{\mathrm{X} \text {, peak }} \sim\right.$ $10^{36-37} \mathrm{erg} \mathrm{s}^{-1}$ ). Two of the eight transients are newly discovered sources, which were both active in 2006 (Swift J174553.7290347 and Swift J174622.1-290634, Degenaar \& Wijnands 2009). Four of the eight transients were observed to recur over the 4-year time span of this campaign (see Table 2) and have relatively short recurrence times. These transients show a different peak flux, duration and lightcurve morphology from outburst to outburst, which is also seen in brighter X-ray transients (Chen et al. 1997).

Currently, there are $13 \mathrm{X}$-ray transients exhibiting 2-10 keV peak luminosities $\gtrsim 10^{34} \mathrm{erg} \mathrm{s}^{-1}$ known in the region covered by the Swift/XRT monitoring observations ${ }^{4}$. Out of these 13 transients, only 1A 1742-289 becomes brighter than $L_{X}>$ $10^{37} \mathrm{erg} \mathrm{s}^{-1}(2-10 \mathrm{keV}$, see Wijnands et al. 2006a, and references therein), while the remaining 12 undergo low-luminosity outbursts. The three sources AX J1745.6-2901, GRS 17412853 and XMM J174457-2850.3 are the brightest amongst these and have $2-10 \mathrm{keV}$ peak luminosities of $10^{36-37} \mathrm{erg} \mathrm{s}^{-1}$, but the other 9 transients have never been observed with luminosities exceeding $10^{36} \mathrm{erg} \mathrm{s}^{-1}$. From the 12 low-luminosity transients, 7 were observed to recur in the past decade and thus have relatively short recurrence times. The remaining 5 objects (CXOGC 174540.0-290031, CXOGC 174554.3285454, XMM J174544-2913.0, Swift J174553.7-290347 and Swift J174622.1-290634) were seen active only once and thus seem to recur less often.

Despite the fact that $>250 \mathrm{ks}$ of new Swift data was obtained, spread over almost daily observations in 2008 and 2009, no new transients were found. Muno et al. (2009) suggested that given the extensive monitoring of the GC in the past years, all X-ray binaries that are located in that region and recur on a timescale of a decade have been identified by now. The galactic population of X-ray binaries (both LMXBs and HMXBs) is expected to encompass 2000 objects (e.g., Verbunt \& van den Heuvel 1995). The region around Sgr A* that has been monitored by Chandra, XMM-Newton and Swift in the past decade covers $\sim 1 \%$ of the stellar mass in the galactic disk (Pfahl et al. 2002).

4 The Swift monitoring observations cover all sources listed in Table A. 1 of Wijnands et al. (2006a) that are located within $\sim 14^{\prime}$ distance from Sgr A*. In addition to the 11 objects from their list, two new transients were discovered by Swift in 2006 (Swift J174553.7-290347 and Swift J174622.1-290634, Degenaar \& Wijnands 2009). 
In this region, $\sim 20$ likely $\mathrm{X}$-ray binaries have been identified (Muno et al. 2009). Most of these are transient sources and strikingly, the majority have very low $2-10 \mathrm{keV}$ peak luminosities of $\lesssim 10^{36} \mathrm{erg} \mathrm{s}^{-1}$ (Muno et al. 2005; Wijnands et al. 2006a; Degenaar \& Wijnands 2009).

The number of likely X-ray binaries that have been identified in the vicinity of $\mathrm{Sgr} \mathrm{A}$ * is thus consistent with that expected from population synthesis models. However, the GC has been monitored with instruments sensitive enough to detect lowluminosity transients only in the past decade and several of the currently known systems appear to have relatively short recurrence times compared to brighter X-ray transients (e.g., Chen et al. 1997). Continued monitoring of the GC is therefore important to search for transient outbursts from new systems to better constrain the number of X-ray binaries located near Sgr A*, and to gain more insight into the duty cycles of known systems.

Acknowledgements. We acknowledge the use of public data from the Swift data archive. This work was supported by the Netherlands Organization for Scientific Research (NWO).

\section{References}

Arnaud, K. A. 1996, in Astronomical Data Analysis Software and Systems V, ed. G. H. Jacoby, \& J. Barnes, ASP Conf. Ser., 101, 17

Burrows, D. N., Hill, J. E., Nousek, J. A., et al. 2005, Space Sci. Rev., 120, 165 Campana, S. 2009, ApJ, 699, 1144

Cannizzo, J. K. 1993, ApJ, 419, 318

Chelovekov, I. V., \& Grebenev, S. A. 2007, Astron. Lett., 33, 807

Chen, W., Shrader, C. R., \& Livio, M. 1997, ApJ, 491, 312

Chenevez, J., Kuulkers, E., Beckmann, V., et al. 2009, The Astronomer's Telegram, 2235

Cocchi, M., Bazzano, A., Natalucci, L., et al. 1999, A\&A, 346, L45

Cooper, R. L., \& Narayan, R. 2007, ApJ, 661, 468

Cornelisse, R., Verbunt, F., in 't Zand, J. J. M., et al. 2002, A\&A, 392, 885

Degenaar, N., \& Wijnands, R. 2009, A\&A, 495, 547

Degenaar, N., Wijnands, R., \& Muno, M. 2008a, The Astronomer's Telegram, 1531

Degenaar, N., Wijnands, R., \& Muno, M. 2008b, The Astronomer's Telegram, 1531

Degenaar, N., Wijnands, R., Kennea, J., \& Gehrels, N. 2010, The Astronomer's Telegram, 2690

Del Santo, M., Sidoli, L., Mereghetti, S., et al. 2007, A\&A, 468, L17

Drilling, J. S., \& Landolt, A. U. 2000, Normal Stars, ed. A. N. Cox, 381

Gehrels, N. 2007, GRB Coordinates Network, 6760

Heinke, C. O., Cohn, H. N., \& Lugger, P. M. 2009, ApJ, 692, 584

in 't Zand, J. J. M., Heise, J., Brinkman, A. C., et al. 1991, Adv. Space Res., 11, 187

in 't Zand, J. J. M., Cumming, A., van der Sluys, M. V., Verbunt, F., \& Pols, O. R. 2005, A\&A, 441, 675

in 't Zand, J. J. M., Jonker, P. G., \& Markwardt, C. B. 2007, A\&A, 465, 953

Kennea, J. A. 2009, The Astronomer's Telegram, 2236

Kennea, J. A., Burrows, D. N., Campana, S., et al. 2006a, The Astronomer's Telegram, 753
Kennea, J. A., \& The Swift/XRT team 2006b, in BAAS, 38, 381

King, A. R., \& Ritter, H. 1998, MNRAS, 293, L42

King, A. R., \& Wijnands, R. 2006, MNRAS, 366, L31

Kuulkers, E., Shaw, S., Chenevez, J., et al. 2007a, The Astronomer's Telegram, 1005

Kuulkers, E., Shaw, S., Chenevez, J., et al. 2007b, The Astronomer's Telegram, 1008

Kuulkers, E., Shaw, S. E., Paizis, A., et al. 2007c, A\&A, 466, 595

Lasota, J.-P. 2001, New Astron. Rev., 45, 449

Lasota, J.-P. 2007, C. R. Phys., 8, 45

Laycock, S., Zhao, P., Torres, M. A. P., et al. 2005, The Astronomer's Telegram, 522

Maeda, Y., Koyama, K., Sakano, M., Takeshima, T., \& Yamauchi, S. 1996, PASJ, 48, 417

Masetti, N., Landi, R., Pretorius, M. L., et al. 2007, A\&A, 470, 331

Mauerhan, J. C., Muno, M. P., Morris, M. R., et al. 2009, ApJ, 703, 30

Menou, K., Esin, A. A., Narayan, R., et al. 1999, ApJ, 520, 276

Muno, M. P., Baganoff, F. K., \& Arabadjis, J. S. 2003a, ApJ, 598, 474

Muno, M. P., Baganoff, F. K., Bautz, M. W., et al. 2003b, ApJ, 589, 225

Muno, M. P., Arabadjis, J. S., Baganoff, F. K., et al. 2004, ApJ, 613, 1179

Muno, M. P., Pfahl, E., Baganoff, F. K., et al. 2005, ApJ, 622, L113

Muno, M. P., Wijnands, R., Wang, Q. D., et al. 2007, The Astronomer's Telegram, 1013

Muno, M. P., Bauer, F. E., Baganoff, F. K., et al. 2009, ApJS, 181, 110

Narayan, R., Garcia, M. R., \& McClintock, J. E. 1997, ApJ, 478, L79

Negueruela, I. 1998, A\&A, 338, 505

Negueruela, I. 2004, in Rev. Mex. Astron. Astrofis. Conf. Ser., ed. G. Tovmassian, \& E. Sion, 20, 55

Negueruela, I., Smith, D. M., Reig, P., Chaty, S., \& Torrejón, J. M. 2006, in The X-ray Universe 2005, ed. A. Wilson, ESA SP, 604, 165

Nespoli, E., Fabregat, J., \& Mennickent, R. E. 2010, A\&A, 516, A94

Nishiyama, S., Nagata, T., Tamura, M., et al. 2008, ApJ, 680, 1174

Peng, F., Brown, E. F., \& Truran, J. W. 2007, ApJ, 654, 1022

Pfahl, E., Rappaport, S., \& Podsiadlowski, P. 2002, ApJ, 571, L37

Ponti, G., Trap, G., Goldwurm, A., et al. 2009, The Astronomer's Telegram, 2038

Porquet, D., Grosso, N., Burwitz, V., et al. 2005, A\&A, 430, L9

Porquet, D., Grosso, N., Goldwurm, A., et al. 2007, The Astronomer's Telegram, 1058

Predehl, P., \& Schmitt, J. H. M. M. 1995, A\&A, 293, 889

Sakano, M., Koyama, K., Murakami, H., Maeda, Y., \& Yamauchi, S. 2002, ApJS, 138,19

Sakano, M., Warwick, R. S., Decourchelle, A., \& Wang, Q. D. 2005, MNRAS, 357,1211

Sidoli, L. 2009, Adv. Space Res., 43, 1464

Sidoli, L., Mereghetti, S., Israel, G. L., et al. 1999, ApJ, 525, 215

Sidoli, L., Romano, P., Mangano, V., et al. 2008, ApJ, 687, 1230

Sunyaev, R. 1990, IAU Circ., 5104

Tokunaga, A. T. 2000, Infrared Astronomy, ed. A. N. Cox, 143

Trap, G., Falanga, M., Goldwurm, A., et al. 2009, A\&A, 504, 501

Verbunt, F., \& van den Heuvel, E. P. J. 1995, in X-ray binaries, ed. W. H. G. Lewin, J. van Paradijs, \& E. P. J. van den Heuvel, 457

Wijnands, R., Rupen, M., Steeghs, D., et al. 2005, The Astronomer's Telegram, 638

Wijnands, R., in 't Zand, J. J. M., Rupen, M., et al. 2006a, A\&A, 449, 1117

Wijnands, R., Kuulkers, E., Muno, M., et al. 2006b, The Astronomer's Telegram, 892

Wijnands, R., Klein-Wolt, M., Kuulkers, E., et al. 2007, The Astronomer's Telegram, 1006

Wijnands, R., Rol, E., Cackett, E., Starling, R. L. C., \& Remillard, R. A. 2009, MNRAS, 393, 126 